\title{
A genome-wide Asian genetic map and ethnic comparison: The GENDISCAN study
}

\author{
Young Seok Ju${ }^{1}$, Hansoo Park ${ }^{1}$, Mi Kyeong Lee ${ }^{2,3}$, Jong-Il Kim¹,2, \\ Joohon Sung ${ }^{3}$, Sung-Il Cho ${ }^{3}$ and Jeong-Sun Seo*1,4
}

Address: ${ }^{1}$ Department of Biochemistry and Molecular Biology, College of Medicine, Seoul National University, Seoul, Korea, ${ }^{2}$ Psoma Therapeutics, Seoul, Korea, ${ }^{3}$ Department of Epidemiology, Graduate School of Public Health and Institute of Health and Environment, Seoul National University, Seoul, Korea and ${ }^{4}$ ILCHUN Genomic Medicine Institute, Medical Research Center, Seoul National University, Seoul, Korea

Email: Young Seok Ju - jueenome@gmail.com; Hansoo Park - hspark27@naver.com; Mi Kyeong Lee - mklee0619@gmail.com; JongIl Kim - jongil@snu.ac.kr; Joohon Sung - jsung@snu.ac.kr; Sung-Il Cho - scho@snu.ac.kr; Jeong-Sun Seo* - jeongsun@snu.ac.kr

* Corresponding author

Published: 25 November 2008

BMC Genomics 2008, 9:554 doi:10.1/86/|47|-2/64-9-554
Received: 6 August 2008

Accepted: 25 November 2008

This article is available from: http://www.biomedcentral.com/I47I-2I64/9/554

(c) 2008 Ju et al; licensee BioMed Central Ltd.

This is an Open Access article distributed under the terms of the Creative Commons Attribution License (http://creativecommons.org/licenses/by/2.0), which permits unrestricted use, distribution, and reproduction in any medium, provided the original work is properly cited.

\begin{abstract}
Background: Genetic maps provide specific positions of genetic markers, which are required for performing genetic studies. Linkage analyses of Asian families have been performed with Caucasian genetic maps, since appropriate genetic maps of Asians were not available. Different ethnic groups may have different recombination rates as a result of genomic variations, which would generate misspecification of the genetic map and reduce the power of linkage analyses.
\end{abstract}

Results: We constructed the genetic map of a Mongolian population in Asia with CRIMAP software. This new map, called the GENDISCAN map, is based on genotype data collected from 1026 individuals of 73 large Mongolian families, and includes 1790 total and 1500 observable meioses. The GENDISCAN map provides sex-averaged and sex-specific genetic positions of 1039 microsatellite markers in Kosambi centimorgans (cM) with physical positions. We also determined $95 \%$ confidence intervals of genetic distances of the adjacent marker intervals.

Genetic lengths of the whole genome, chromosomes and adjacent marker intervals are compared with those of Rutgers Map v.2, which was constructed based on Caucasian populations (Centre d'Etudes du Polymorphisme Humain (CEPH) and Icelandic families) by mapping methods identical to those of the GENDISCAN map, CRIMAP software and the Kosambi map function. Mongolians showed approximately 1.9 fewer recombinations per meiosis than Caucasians. As a result, genetic lengths of the whole genome and chromosomes of the GENDISCAN map are shorter than those of Rutgers Map v.2. Thirty-eight marker intervals differed significantly between the Mongolian and Caucasian genetic maps.

Conclusion: The new GENDISCAN map is applicable to the genetic study of Asian populations. Differences in the genetic distances between the GENDISCAN and Caucasian maps could facilitate elucidation of genomic variations between different ethnic groups. 


\section{Background}

Genetic maps provide specific positions of genetic markers, which are required for performing genetic studies. Linkage analyses, which aim to identify genetic loci related to human phenotypes and complex diseases, have been performed with Caucasian genetic maps even in Asian populations, because no comprehensive Asian genetic maps with dense markers have yet been introduced. Since multipoint methods are frequently used in linkage analyses, it is important to use correct maps for the population being studied [1].

Distance between adjacent genetic markers in genetic maps is calculated from average recombination rates between markers during meiosis with map functions. The Kosambi map function is widely used nowadays.

Genetic mapping was first introduced using restriction fragment length polymorphism (RFLP) markers [2], followed by genome-wide human genetic maps with more informative microsatellite markers. The Genethon [3] and Marshfield [4] maps were created from eight CEPH families [5], using 5264 and 8325 genetic markers, respectively, but few (< 190) meioses. The deCODE map utilized 1257 meioses in 146 Icelandic families with 5136 markers [6]. In addition, several combined genetic maps were generated based on the CEPH and Icelandic populations [7-10].

Other than for Caucasians, however, there are few human genetic maps for different ethnic groups. Although genetic maps of four different ethnic groups (African Americans, Mexican Americans, East Asians, and Whites) were recently constructed, the number of markers was quite small $(n=353)$ and the maps were constructed based on nuclear families [11]. Misspecification of genetic maps may reduce the power of linkage analyses $[1,12]$, and different ethnic groups may have different recombination rates [13]. Therefore, separate genetic maps for Asian populations are needed to investigate the Asian genome more precisely.

We constructed an Asian genetic map with 1039 microsatellite markers using 1026 genotyped individuals in 73 large Mongolian families. This study was undertaken as a part of GENDISCAN (GENe DIScovery for Complex traits in isolated large families of Asians of Northeast) project. The construction of an Asian genetic map may be applicable to further linkage studies of Asian ethnic groups as well as to understanding the genomic variations between Asians and Caucasians with megabase resolution.

\section{Results}

Files providing details of the GENDISCAN map (e.g., the genetic/physical positions, the genetic distance of inter- vals, the 95\% confidence intervals of the genetic distance of intervals, the genetic distance of Rutgers Map v. 2 intervals, the p-values denoting the significance levels for differences in the genetic distance between the GENDISCAN map and Rutgers Map v.2 intervals, the heterozygosity of markers, the number of informative meioses of markers and the number of informative meioses between markers) are available. See additional file 1: Details of GENDISCAN map.

We genotyped 73 families, consisting of 1446 family members and a total of 1790 meioses. Among the 1446 family members, 1026 were genotyped and 1500 meioses became available for investigation. Considering the heterozygosity of the 1039 microsatellite markers genotyped in this study, 47 to 1098 informative meioses of each marker (average, 711.5) were obtained using CRIMAP software [14]. Only 18 markers (1.7\%) showed fewer than 400 informative meioses.

The GENDISCAN map shows the genetic positions of the markers, both sex-averaged and sex-specific, along with the physical positions. A summary of the map is presented in Table 1. The physical lengths of the chromosomes are also included. The GENDISCAN map covers 2703.1 Mb, which is $94.3 \%$ of the human genome assembly Build 36.2. When we excluded the telomeric heterochromatic regions, which have a wide range of sequencing gaps in especially acrocentric chromosomes $(13,14,15,21,22)$, the coverage increases to $96.9 \%$.

We compared our GENDISCAN map with the Rutgers Map v.2, one of the most accurate genetic maps of Caucasians (generated from CEPH and Icelandic families) and including 28121 polymorphic markers, with an average of 301 informative meioses [8]. Among the 1039 markers shown in the GENDISCAN map, we were able to determine the genetic positions of 1006 microsatellite markers common to both the GENDISCAN and Rutgers Map v.2, from Rutgers Map v.2. The genetic positions of the remaining 33 markers, which are not present in Rutgers Map v.2, were estimated by an interpolation considering the physical positions of the markers from human genome assembly Build 36.2.

The sex-averaged, female and male whole genome lengths were $3230.6 \mathrm{cM}, 3906.0 \mathrm{cM}$ and $2394.0 \mathrm{cM}$, respectively, which are $5-9 \%$ shorter than the respective genome lengths of Rutgers Map v.2 (Figure 1). The genetic lengths of chromosomes of the sex-averaged, female and male maps are illustrated in Figures 2, 3 and 4, respectively. All chromosomal lengths of the GENDISCAN map were shorter than those of the Rutgers Map v.2 except for male chromosomes 3 and 6, and sex-averaged chromosome 3 . Paired t-tests demonstrated significant differences 
Table I: Summary of the GENDISCAN genetic map

\begin{tabular}{|c|c|c|c|c|c|}
\hline \multirow[b]{2}{*}{ Chromosome } & \multirow[b]{2}{*}{ Physical length (Mb) ${ }^{\mathrm{a}}$} & \multicolumn{3}{|c|}{ Genetic length (cM) } & \multirow[b]{2}{*}{ Number of markers } \\
\hline & & Sex-averaged & Female & Male & \\
\hline 1 & 237.49 & 250.06 & 308.57 & 174.69 & 78 \\
\hline 2 & 241.18 & 244.91 & 302.42 & 169.86 & 77 \\
\hline 3 & 198.27 & 222.46 & 265.95 & 168.87 & 63 \\
\hline 4 & 189.65 & 202.05 & 252.60 & 138.39 & 59 \\
\hline 5 & $|78.7|$ & 195.36 & 236.40 & | 44.54 & 63 \\
\hline 6 & 168.57 & 184.24 & 226.30 & 131.55 & 54 \\
\hline 7 & 156.34 & 168.64 & 206.52 & II8.44 & 62 \\
\hline 8 & 138.60 & 149.42 & 192.26 & 97.04 & 53 \\
\hline 9 & 139.63 & 157.76 & 183.52 & 127.62 & 51 \\
\hline 10 & 134.03 & 171.84 & 207.56 & 128.48 & 49 \\
\hline 11 & 131.40 & 145.60 & 178.38 & 105.82 & 46 \\
\hline 12 & 131.30 & 165.69 & 196.21 & 127.66 & 51 \\
\hline 13 & 93.58 & 126.13 & 152.58 & 94.45 & 33 \\
\hline 14 & 83.70 & 109.29 & 121.54 & 94.15 & 44 \\
\hline 15 & 74.73 & 103.49 & 124.86 & 77.28 & 40 \\
\hline 16 & 86.50 & 117.80 & 137.95 & 94.00 & 36 \\
\hline 17 & 75.04 & 123.30 & 148.84 & 93.59 & 43 \\
\hline 18 & 71.35 & 108.47 & 130.88 & 80.07 & 33 \\
\hline 19 & 61.41 & 99.17 & 111.57 & 85.52 & 32 \\
\hline 20 & 56.64 & 88.53 & 104.83 & 70.55 & 31 \\
\hline 21 & 26.18 & 44.30 & 55.99 & 29.47 & 20 \\
\hline 22 & 28.81 & 52.08 & 60.25 & 41.96 & 21 \\
\hline Total & 2703.11 & 3230.59 & 3905.98 & 2394.00 & 1039 \\
\hline
\end{tabular}

a Physical length from the first to the last marker of each chromosome of the GENDISCAN map; marker positions are from human genome assembly Build 36.2

between the whole genome and chromosome genetic lengths (Table 2). The whole genome and chromosome 2

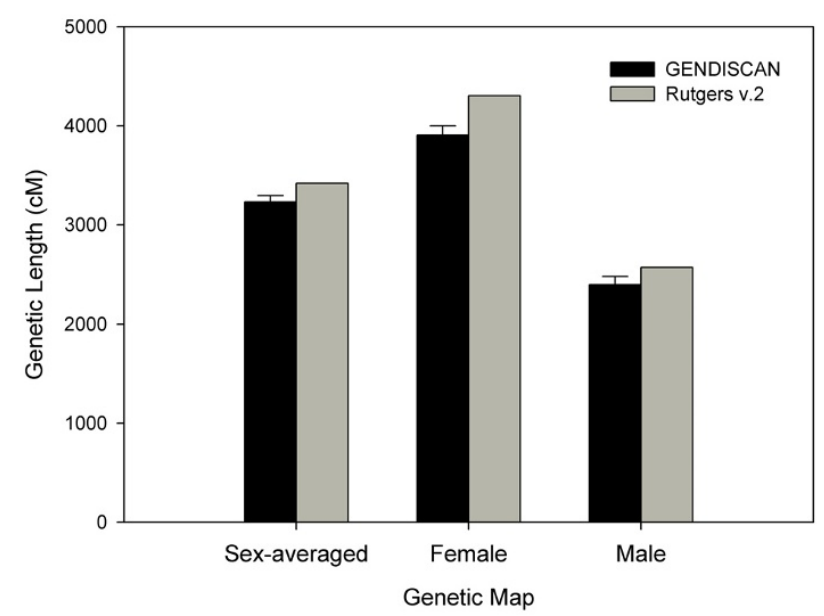

Figure I

Comparison of whole genome lengths between the GENDISCAN map and Rutgers Map v.2. Error bars represents the $95 \%$ confidence intervals from the paired $t$ tests. lengths of all three types of GENDISCAN map were significantly shorter than those of the Rutgers MAP v.2.

The genetic distances between adjacent markers and the $95 \%$ confidence intervals were estimated. The average intermarker spacing was $2.66 \mathrm{Mb}$ and $3.17 \mathrm{cM}$. The inter-

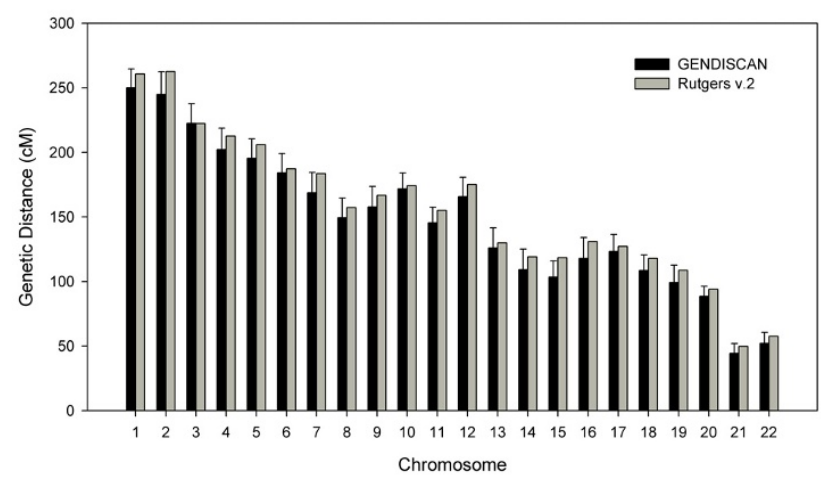

Figure 2

Comparison of sex-averaged genetic lengths of the chromosomes between the GENDISCAN map and Rutgers Map v.2. Error bars represents the $95 \%$ confidence intervals from the paired $t$-tests. 


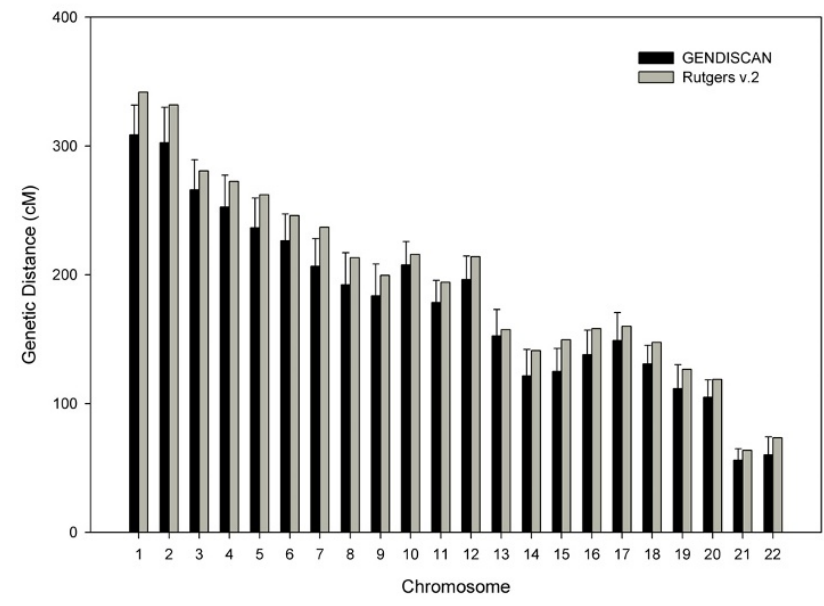

Figure 3

Comparison of female genetic lengths of the chromosomes between the GENDISCAN map and Rutgers Map v.2. Error bars represents the $95 \%$ confidence intervals from the paired t-tests.

marker recombination rate was derived by dividing the intermarker genetic distance by the physical distance. The recombination rate patterns for chromosomes are illustrated in additional files (see additional files 2, 3, and 4: recombination rates of the sex-averaged, female and male maps, respectively). These figures are helpful in comparing the genome-wide recombination patterns of GENDISCAN and Rutgers Map v.2. The sex-averaged recombination rate patterns of chromosome $8 \mathrm{p}$ were quite different (Figure 5). We compared 1017 intermarker genetic distances between GENDISCAN and Rutgers Map v.2, and calculated p-values for the significance of these differences (Figure 6, see Methods). A histogram of these

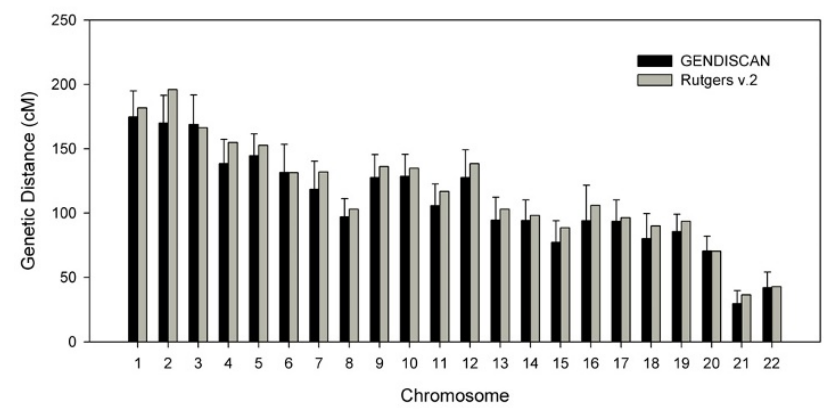

Figure 4

Comparison of male genetic lengths of the chromosomes between the GENDISCAN map and Rutgers Map v.2. Error bars represents the $95 \%$ confidence intervals from the paired t-tests.
1017 normalized intermarker-interval-differences, or $\mathrm{z}$ scores transformed from the corresponding p-values, showed that the distribution of intermarker-interval-differences between the GENDISCAN map and Rutgers Map v. 2 was close to normal (Figure 7).

Although most of the intervals of the GENDISCAN map and Rutgers Map v. 2 were in good agreement, 40 intervals (3.9\%) differed significantly after Bonferroni's multiple comparison correction ( $\mathrm{p}$-value $<4.9 \times 10-5$ ). Two of these 40 intervals were excluded, since their intermarker genetic distances on Rutgers Map v.2 were derived by interpolation. Thus, we identified 38 ethnically different marker intervals (Table 3). The differences in local genomic structure difference in these intervals may cause local recombination rate differences among ethnic groups.

\section{Discussion}

The human genome varies among ethnic groups as a result of their diverse history. Complex phenotypes result from the interaction of different genes with the unique environments to which humans are exposed. Finding specific disease loci within families helps identify the genetic causes of complex diseases effectively.

The GENDISCAN study, which began in 2003, was designed to identify specific genetic loci and genes that influence complex traits and diseases in Northeast Asian populations. As the lifestyle of Northeast Asians has become more westernized, the prevalence of complex diseases, such as diabetes, obesity, cardiovascular diseases and cancers, has increased. Linkage analyses require appropriate genetic maps for identifying correct loci. Hence we constructed a genetic map of an Asian population as an initial step in our GENDISCAN study.

World populations can be grouped into nine clusters based on genetic distances: African; New Guinean and Australian; Pacific Islander; Southeast Asian; Northeast Asian; Arctic Northeast Asian; Amerind; North African and West Asian; and European [15,16]. The Northeast Asian cluster includes Japanese, North Chinese, Koreans and Mongolians. Since genetic distance within clusters is closer than between clusters, our genetic map of Mongolians may be more applicable to Japanese, North Chinese and Koreans than genetic maps of Caucasians.

When we compared the GENDISCAN map with the Caucasian Rutgers Map v.2, we found that the genetic length of the GENDISCAN map was much shorter. Genomewide, Mongolians show about 1.9 fewer recombinations per meiosis compared with Caucasians. This is due to a general trend of 1017 marker intervals overall rather than several specific genomic regions. Although the genome- 
Table 2: Comparison of genetic map distances of the GENDISCAN map and Rutgers Map v.2

\begin{tabular}{|c|c|c|c|c|c|c|c|}
\hline \multirow[b]{2}{*}{ Chr } & \multirow[b]{2}{*}{ Number of intervals } & \multicolumn{2}{|c|}{ Sex-averaged map } & \multicolumn{2}{|c|}{ Female map } & \multicolumn{2}{|c|}{ Male map } \\
\hline & & $\underset{\mathrm{a}}{\text { Length Difference (cM) }}$ & $\mathrm{p}$-value ${ }^{b}$ & $\underset{\mathrm{a}}{\text { Length Difference (cM) }}$ & $\mathrm{P}$-value ${ }^{\mathrm{b}}$ & $\begin{array}{l}\text { Length Difference } \\
(\mathrm{cM})^{\mathrm{a}}\end{array}$ & $\mathrm{P}$-value ${ }^{\mathrm{b}}$ \\
\hline 1 & 77 & -10.64 & 0.150 & -33.12 & $0.005 * *$ & -7.00 & 0.491 \\
\hline 2 & 76 & -17.80 & $0.048 *$ & -29.36 & $0.037 *$ & -26.12 & $0.019 *$ \\
\hline 3 & 62 & 0.14 & 0.985 & $-|4.5|$ & 0.219 & 2.43 & 0.832 \\
\hline 4 & 58 & -10.56 & 0.214 & -19.84 & 0.114 & -16.48 & 0.085 \\
\hline 5 & 62 & -10.55 & 0.166 & -25.66 & $0.030 *$ & -8.16 & 0.342 \\
\hline 6 & 53 & -3.06 & 0.684 & -19.67 & 0.062 & 0.16 & 0.988 \\
\hline 7 & 61 & -14.80 & 0.067 & -30.30 & $0.007 * *$ & -13.42 & 0.227 \\
\hline 8 & 52 & -7.76 & 0.311 & -20.92 & 0.097 & -5.97 & 0.405 \\
\hline 9 & 50 & -9.02 & 0.259 & -16.01 & 0.200 & -8.50 & $0.34 I$ \\
\hline 10 & 48 & -2.35 & 0.700 & -8.24 & 0.367 & -6.35 & 0.459 \\
\hline 11 & 45 & -9.54 & 0.113 & -15.86 & 0.069 & -10.99 & 0.192 \\
\hline 12 & 50 & -9.53 & 0.209 & -17.87 & 0.056 & -10.82 & 0.316 \\
\hline 13 & 32 & -3.73 & 0.624 & -4.73 & $0.64 I$ & -8.46 & 0.342 \\
\hline 14 & 43 & -9.92 & 0.211 & -19.53 & 0.060 & -4.05 & 0.613 \\
\hline 15 & 39 & -14.99 & $0.019 *$ & -24.53 & $0.009 * *$ & -11.23 & 0.183 \\
\hline 16 & 35 & $-|3.2|$ & 0.110 & -20.16 & $0.038 *$ & -12.14 & 0.378 \\
\hline 17 & 42 & -3.90 & 0.554 & -11.11 & 0.311 & -2.88 & 0.728 \\
\hline 18 & 32 & -9.44 & 0.120 & -16.62 & $0.024 *$ & -9.89 & 0.308 \\
\hline 19 & 31 & -9.60 & 0.157 & -15.06 & 0.107 & -8.06 & 0.233 \\
\hline 20 & 30 & -5.52 & 0.160 & -14.03 & $0.043 *$ & 0.26 & 0.963 \\
\hline 21 & 19 & -5.34 & 0.158 & -7.62 & 0.089 & -6.97 & 0.168 \\
\hline 22 & 20 & -5.57 & 0.191 & -13.09 & 0.062 & -0.89 & 0.880 \\
\hline Total & 1017 & -186.69 & $1 \times 10^{-8} 8 * *$ & -397.84 & $8 \times 10^{-17} * * *$ & -175.53 & $4 \times 10^{-5} * * *$ \\
\hline
\end{tabular}

aRutgers Map v.2 genetic length subtracted from that of the GENDISCAN map.

$\mathrm{b} p$-values estimated from paired t-tests (two-sided).

$* p<0.05$, ** $p<0.01$, *** $p<0.001$.

Chr; Chromosome

wide recombination rate patterns showed good agreement between the two ethnic groups, those of Asians were generally smaller. However, we also identified several regions in which the patterns did not correlate; that is some recombination jungles in the GENDISCAN map appear as recombination deserts in Rutgers Map v.2, and vice versa.

A previous examination of ethnic differences in genetic maps identified no significant differences for genomewide genetic length between Caucasians and Asians, but found a significant local difference on $8 \mathrm{p}$, a finding identical to ours [11]. The ethnic difference on $8 p$ is likely due to a frequent local polymorphic inversion [11]. Interestingly, we found a suggestive inversion of marker orders against the physical map on 8p22 (D8S520 and D8S1759 with likelihood of [inversion]/[original] $=17.8$ ) by FLIPS option of CRIMAP. The sex averaged map of chromosome 8 , reflecting the inversion, is available on additional file 5 . Since previous ethnic-specific maps were constructed using a small number of genetic markers $(\mathrm{n}=353)$ and nuclear families, with most families made up of no grandparents and several children, these findings may be less robust than ours [11]. Generally, determining the phase of genotypes to find recombinations requires genetic information of three generations, or that of two generations with many children.

Recombination is related to diversity of DNA sequences, linkage disequilibrium (LD) and copy number changes [17]. Asian-Americans have a smaller number of single nucleotide polymorphisms (SNPs) than European-Americans (5050 versus 6736) and lower minor allele frequencies (MAFs) of the SNPs (820 (16\%) versus $1579(23 \%)$ whose MAFs > 5\%) [18]. Moreover, Asians have a smaller number of haplotypes per block than Caucasians (3.5 versus 4.2) [19] and Asian Americans have fewer copy number variations (CNVs) than European Americans (14.8 versus 16.3) [20]. These findings indicate that Asians have a more homogeneous genome than Caucasians, probably as a result of their low recombination rate.

Although genetic studies using SNP markers or resequencing on random populations rather than families give finescale recombination pattern data, these data are indirect and may be biased by mutation, selection, drift and demography [21]. Most recombinations occur in short kilobase scale regions, known as recombination hotspots 


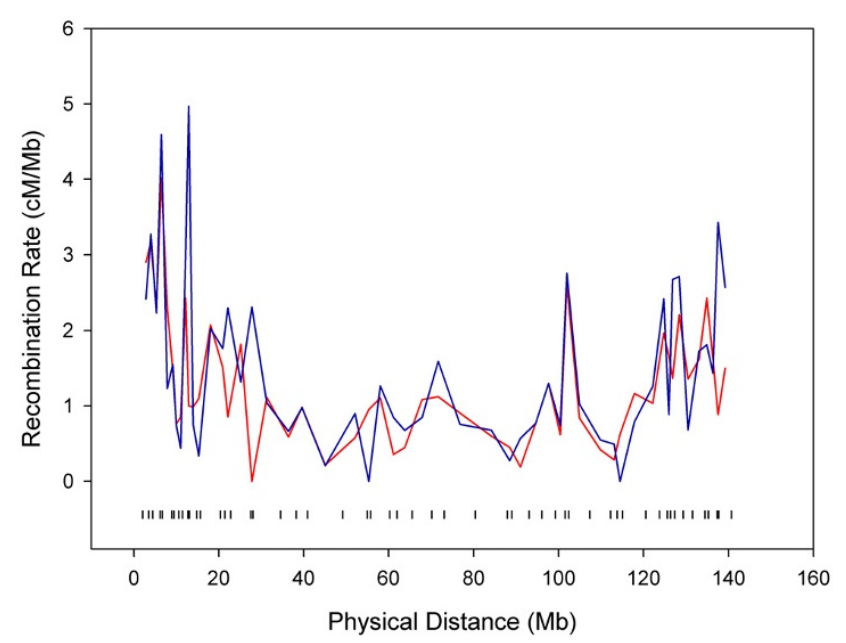

Figure 5

Comparison of sex-averaged recombination rates of chromosome 8 between the GENDISCAN map (red line) and Rutgers Map v.2 (blue line). The physical positions of genetic markers are denoted by black vertical marks.

[22]. Fine-scale data have suggested that these hotspots and intensities may differ among different ethnic groups [23]. Moreover, population-specific hotspots have also been identified, and populations of close geographic

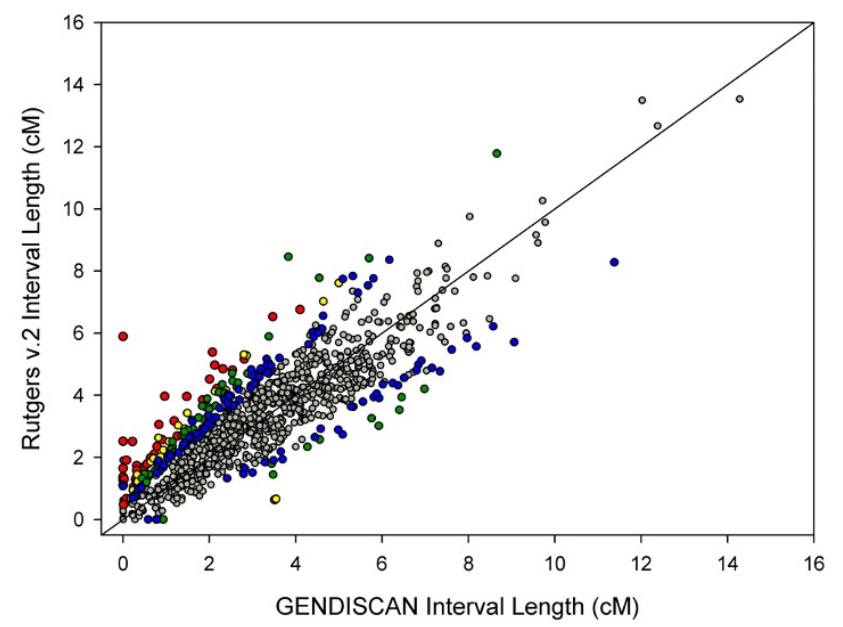

Figure 6

Comparison of genetic distances of 1017 marker intervals between the GENDISCAN map and Rutgers Map v.2. Color of dots represents the p-values for significances of genetic length differences between the GENDISCAN map and Rutgers Map v.2. Red, $p<0.000$ I; yellow, $p<0.00$ I; green, $p<0.0$ I; blue, $p<0.05$ and gray, $p$ $>0.05$

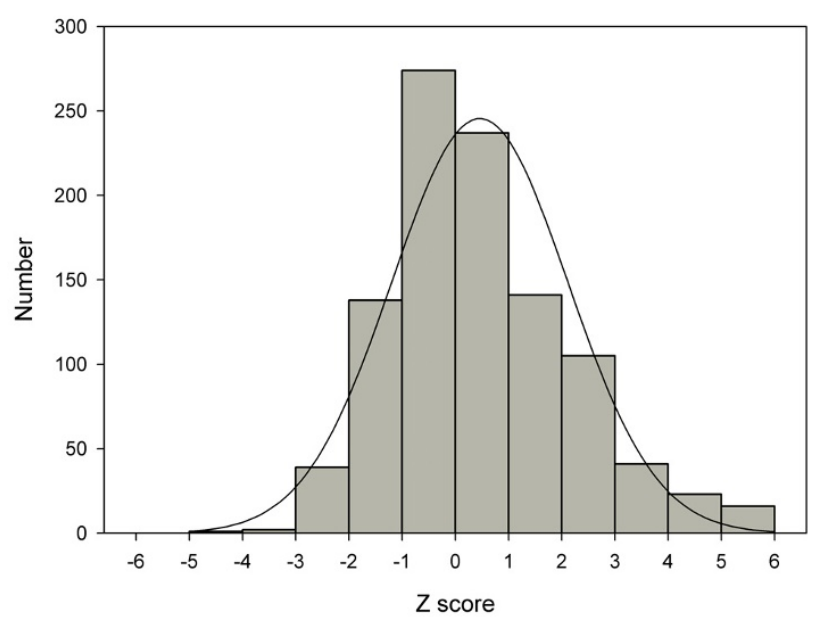

Figure 7

Distribution of intermarker interval differences between GENDISCAN map and Rutgers Map v.2. A histogram of 1017 normalized intermarker-interval-differences. The $z$ scores are transformed from the $p$-values representing the significance of intermarker-interval-differences between the GENDISCAN map and Rutgers Map v.2. A curve representing normal distribution is included for comparison.

regions tend to show similar hotspot intensities [24]. The properties of recombination hotspots are not well known, but some characteristics have been described [22]. The local DNA sequences of recombination hotspots present more long terminal repeats of retrotransposons, THE1A and THE1B, as well as CT-rich and GA-rich repeats [22]. Some DNA motifs, such as the CCTCCCT oligomer of THE1A and THE1B, and the CCCCACCCC oligomer within recombination hotspots, may be local DNA signals of recombination hotspots [22]. Additional studies of recombination patterns, with comparisons among different ethnic groups, are necessary to understand the nature of recombination hotspots. The present study, which found significantly different marker intervals between two ethnic groups, can facilitate further comparisons of genomic variations among ethnic groups.

Some comparative characteristics of the GENDISCAN map and Rutgers Map v.2 deserve attention. The number of markers used for genetic mapping was quite different (1039 for GENDISCAN map, 23389 for autosomes of Rutgers Map v.2). This may have caused the difference in genetic distances, since high, but not low, density markers can identify two very close individual recombination events. These double recombination events, however, were eliminated when constructing both maps, since they were thought likely to be non-Mendelian genotyping 
Table 3: List of marker intervals that differed significantly (two-sided $p<4.9 \times 10^{-5}$ ) between the GENDISCAN and Rutgers v.2 sex averaged maps

\begin{tabular}{|c|c|c|c|c|}
\hline Cytogenetic Position & Marker Interval & GENDISCAN Interval Length & Rutgers v.2 Interval Length & P-value \\
\hline Ip34.3 & DIS255-DISI86 & 0.47 & 1.4 & $6 \times 10^{-6}$ \\
\hline $2 p 25.3$ & D2S323-D2S319 & 0.83 & 3.06 & $9 \times 10^{-8}$ \\
\hline $2 p 16.1$ & D2SI364-D2S370 & 0.59 & 1.82 & $2 \times 10^{-6}$ \\
\hline $2 p|4-p| 3.2$ & $\mathrm{D} 2 \mathrm{~S} 2152-\mathrm{D} 2 \mathrm{~S} 2110$ & 2.12 & 4.97 & $7 \times 10^{-8}$ \\
\hline $2 q 33.3$ & D2S2358-D2S232I & 0 & 1.65 & $8 \times 10^{-10}$ \\
\hline $2 q 37.2$ & D2S2973-D2S2202 & 0.63 & 1.99 & $3 \times 10^{-5}$ \\
\hline $3 q 29$ & D3S240-D3SI 265 & 0 & 2.52 & 0 \\
\hline $4 q 33-q 34.1$ & D4S2910-D4S1539 & 0.97 & 3.97 & $2 \times 10^{-14}$ \\
\hline $5 q 14.3-q 15$ & D5SI725-D5S2498 & 1.25 & 2.69 & $2 \times 10^{-5}$ \\
\hline $5 q 21.1$ & D5SI503-D5S409 & 0.22 & 2.51 & 0 \\
\hline $5 q 22.1$ & D5S250I-D5S2027 & 0.56 & 1.79 & $6 \times 10^{-6}$ \\
\hline $6 p 21.31-p 21.1$ & D6SI576-D6SI575 & 4.10 & 6.76 & $2 \times 10^{-5}$ \\
\hline $6 q 21$ & D6S4I6-D6S1603 & 0.03 & 1.30 & 0 \\
\hline $8 p 22$ & D8S552-D8SI790 & 0.32 & 1.59 & $2 \times 10^{-8}$ \\
\hline $8 p 21.3$ & D8SIII6-D8SI734 & 1.19 & 3.17 & $6 \times 10^{-6}$ \\
\hline $8 q 21.3$ & D8S273-D8S270 & 0.79 & 2.35 & $2 \times 10^{-6}$ \\
\hline $8 q 24.23$ & D8SI783-D8S274 & 0.31 & 1.20 & $2 \times 10^{-5}$ \\
\hline $9 q 22.2-q 22.31$ & D9S283-D9SI78I & 2.17 & 4.22 & $9 \times 10^{-6}$ \\
\hline $9 q 31.2$ & D9SII62-D9S26I & 0.08 & 1.91 & 0 \\
\hline IOpI5.3 & DIOS249-DI0S602 & 2.07 & 5.39 & $1 \times 10^{-6}$ \\
\hline||$q \mid 4.2$ & DIISI887-DIISI780 & 0.50 & 1.71 & $2 \times 10^{-6}$ \\
\hline $12 \mathrm{q} 23.3$ & DI2SI636-DI2SI683 & 0.07 & 0.67 & $4 \times 10^{-7}$ \\
\hline $13 q 33.3-q 34$ & $D|3 S 778-D| 3 S|3| 5$ & 2.80 & 5.16 & $2 \times 10^{-5}$ \\
\hline $13 q 34$ & DI3S26I-DI3S285 & 0.91 & 2.57 & $9 \times 10^{-8}$ \\
\hline$|4 q| 1.2$ & DI4S283-DI4S990 & 3.51 & 0.64 & $1 \times 10^{-5}$ \\
\hline$|4 q| 2-q \mid 3.1$ & DI4SI07I-DI4S74I & 3.47 & 6.53 & $1 \times 10^{-6}$ \\
\hline $15 q|2-q| 3.1$ & DI5SI56-DI5SI0I9 & 2.00 & 4.52 & $1 \times 10^{-6}$ \\
\hline $15 q 15.1$ & DI5SI46-DI5S2I4 & 0.01 & 0.48 & 0 \\
\hline $15 q 26.2$ & DI5SI0I4-DI5S2I2 & 0.48 & 1.56 & $5 \times 10^{-6}$ \\
\hline $16 p \mid 3.3$ & DI6S52I-DI6S3395 & 0 & 5.89 & 0 \\
\hline $16 p \mid 3.2$ & DI6S3087-DI6S404 & 0.25 & 1.05 & $2 \times 10^{-6}$ \\
\hline$|7 p| 3.2$ & DI7SI854-DI7SI832 & 0.72 & 2.10 & $1 \times 10^{-5}$ \\
\hline $17 q 25.1-q 25.3$ & DI7S2192-DI7S802 & 2.54 & 4.81 & $8 \times 10^{-6}$ \\
\hline$|8 p| 1.3 \mid$ & DI8SII32-DI8S452 & 1.48 & 3.96 & $1 \times 10^{-6}$ \\
\hline $18 q 21.33$ & DI8SII47-DI8S68 & 1.84 & 3.85 & $7 \times 10^{-6}$ \\
\hline $19 q 13.3$ & DI9S59I-DI9S424 & 0.29 & 1.30 & $3 \times 10^{-8}$ \\
\hline $21 q 21.1$ & D2IS1902-D2ISI884 & 0.32 & 1.74 & $3 \times 10^{-13}$ \\
\hline $2 \operatorname{lq} 21.2$ & D2IS272-D2ISI9|4 & 0 & 1.37 & $3 \times 10^{-14}$ \\
\hline
\end{tabular}

errors, not two individual recombinations. Compared with Rutgers Map v.1, although the number of markers used in Rutgers Map v.2 was increased nearly twofold $[7,8]$, the marker-matched genome-wide genetic length of Rutgers Map v. 2 is $2 \mathrm{cM}$ shorter than that of Rutgers Map v.1 (data not shown). Moreover, construction of the GENDISCAN genetic map of chromosome 1 using fewer and fewer markers increases, rather than decreases, the genetic length (data not shown). Biologically, the double recombinations are considered very rare in human meiosis, since not only is recombination uncommon (about 32 per genome per meiosis), but also one chiasma inhibits formation of another chiasma nearby (positive interference). The Kosambi map function, which is widely used and thought to reflect adequate levels of double recombination in humans, has been applied for calibrating the slight possibility of double recombination when constructing genetic maps [25]. Therefore, if non-Mendelian genotyping errors, which appear as double recombinations, were properly eliminated during the cleaning process, there is no reason to expect that small numbers of markers reduce genetic length in genetic maps.

The statistical methods used in the comparisons are also worthy of note. The paired t-test, which is used to compare of genetic lengths of the whole genome and chromosomes, is not the method of choice for testing the difference between sums of intervals (genetic lengths of the whole genome and chromosomes), but is the method of choice for testing the difference between the average of intervals. However, since each interval distance of the GENDISCAN map is significantly shorter than that of Rut- 
gers Map v.2, the sum of interval distances of GENDISCAN is likely shorter. We therefore used paired t-tests to compare of whole genome genetic lengths and to estimate the significance levels of their differences.

We assumed that the number of recombination events between markers would follow a binomial distribution, then a normal distribution for estimating the 95\% confidence interval of each intermarker genetic distance (see Methods, Statistical Methods for details). Adjusted Wald methods were used for marker intervals, whose $\theta$ equals zero. The 95\% confidence intervals of the GENDISCAN interval distances and p-values for the significance of differences between GENDISCAN and Rutgers Map v.2 intervals must be interpreted with caution, since they were calculated under those assumptions. However, we believe that those values are helpful parameters for assessing the certainty and finding the significant differences between genetic maps.

\section{Conclusion}

In summary, we constructed a genetic map with large Asian families. The GENDISCAN map may provide better results than Caucasian genetic maps in linkage analysis of Asians. We also found that the GENDISCAN map shows shorter genetic distances than a Caucasian genetic map, with Asians having 1.9 fewer recombination events per meiosis than Caucasians. The recombination rates of some marker intervals differed significantly between populations. Our results illustrate the differences in recombination patterns between ethnic groups and provide clues to their underlying genomic variations.

\section{Methods \\ Subjects}

Genetic mapping was performed as part of the GENDISCAN study, designed for linkage analysis of a number of complex traits of the Asian population. In 2006, we collected and genotyped 978 individuals in Dashbalbar, Dornod Province, Mongolia. Their relationships were determined from interviews and confirmed by genotype data (see Methods, Genotyping). Informed consent was obtained from all enrolled subjects, and the study protocol was approved by the institutional review board (IRB) of Seoul National University (approval number, H-0307105-002).

The pedigree size was exceedingly large for an effective analysis; it included seven families, with the largest including 949 genotyped subjects. Hence, we separated the seven families into 73 families, causing 44 genotyped subjects to be included in more than one family. However, no meioses overlapped in this procedure. The final pedigree included 1446 individuals, with 1026 subjects genotyped, and a total of 1790 meioses, with 895 for each gender, and 1500 meioses available for investigation.

\section{Genotyping}

Venous blood was collected and DNA was extracted from leukocytes using standard protocols. Genotyping was completed with 1039 microsatellite markers throughout the autosomes by deCODE genetics.

Genotyping errors were detected and removed using three software packages: nonpaternity was checked with PREST [26]; individual relationships other than paternity were identified and corrected with PEDCHECK [27]; and nonMendelian errors were investigated with SimWalk [28]. Mendelian and non-Mendelian errors constituted $0.13 \%$ and $0.26 \%$, respectively, of all genotype data.

\section{Genetic Mapping}

After correcting genotype errors, the GENDISCAN map was generated with CRIMAP software [14]. The orders of markers were determined by comparison with the physical map of the human genome assembly Build 36.2. We confirmed the order of markers with the FLIPS option. The FIXED option of CRIMAP was used to calculate recombination fractions between two successive markers. The Kosambi map function was used for the estimation of genetic distances. Genetic maps for both the sex specific and sex averaged genetic maps were generated.

\section{Statistical Methods}

The genetic lengths of the whole genome and each chromosome were compared between the GENDISCAN and Rutgers Map v.2 using paired t-tests. For these comparisons, all 1017 intermarker intervals contributed to genome-wide genetic distance estimation and the marker intervals on the particular chromosome to the genetic distance of each chromosome.

To estimate the 95\% confidence interval of each intermarker genetic distance of the GENDISCAN map, we assumed that the number of recombination events between adjacent markers would follow a binomial distribution $(N r \sim \mathrm{B}(\mathrm{Nm}, \theta): \mathrm{Nr}$, the number of recombination; $N m$, the number of informative meioses; $\theta$, recombination fraction). Moreover, such a distribution was transformed into a normal distribution, since the number of meioses between markers was not small $(n>30)$. We obtained $\theta$ and $\mathrm{Nm}$ of each intermarker interval from the CRIMAP software [14], which calculates recombination fractions through both two-point and multipoint maximum likelihood estimation. For example, if M1, M2, M3 and M4 are genetic markers with the correct order in a 
pedigree, the CRIMAP software estimates $\theta$ between M2 and $M 3$, even if the interval $(M 2, M 3)$ is not informative, using the flanking informative markers (M1 and M4). Therefore, the $\mathrm{Nm}$ obtained above is not the direct value for calculating the final recombination fraction, but was used to understand approximately how large the $\mathrm{Nm}$ was that was used for estimating $\theta$.

The final estimates of $\theta$ and $N m$ for each interval were used to calculate the $95 \%$ confidence intervals of recombination fractions using the following equations:

$$
\theta \pm 1.959 \cdot \mathrm{S}_{\theta} / \sqrt{ } N m \text {, where } \mathrm{S}_{\theta}^{2}=\theta \cdot(1-\theta),
$$

which are from the normal distribution and binomial distribution, respectively. In the case where the estimated $\theta$ equals 0 , we applied the adjusted Wald method, in which $\theta$ is replaced by $\theta=2 /(\mathrm{Nm}+4)$ [8]. In this study, 25 of the 1017 intermarker recombination fractions were calculated by adjusted Wald methods. Finally, the 1017 recombination fractions and the confidence intervals were transformed into genetic distances (cM) by the Kosambi map function. In addition, the statistical significance levels of differences in $\theta$ s between GENDISCAN and Rutgers Map v. 2 were tested based on the confidence intervals. Bonferroni's multiple comparison correction method was applied to determine significant differences ( $\mathrm{p}$-value $<4.9$ $\left.\times 10^{-5}\right)$.

\section{Authors' contributions}

YSJ designed the study, carried out the genetic mapping, performed statistical analyses, and drafted the manuscript. HP participated in the survey and genotyping and helped to draft the manuscript. MKL managed the pedigree and genotype data of subjects. JIK participated in the design and coordination of the study. JS and SIC helped to perform the statistical analyses and to draft the manuscript. JSS participated in the study design and coordination and helped to draft the manuscript. JIK, JS, SIC, and JSS conceived of the whole GENDISCAN study. All authors read and approved the final manuscript.

\section{Additional material}

\section{Additional file 1}

Details of the GENDISCAN map. A file providing details of the GENDISCAN map (e.g., the genetic/physical positions, the genetic distance of intervals, the $95 \%$ confidence intervals of the genetic distance of intervals, the genetic distances of Rutgers Map v.2 intervals, the p-values denoting the significance levels for differences in the genetic distance between the GENDISCAN map and Rutgers Map v.2 intervals, the heterozygosity of markers, the number of informative meioses of markers and the number of informative meioses of between markers).

Click here for file

[http://www.biomedcentral.com/content/supplementary/14712164-9-554-S1.xls]

\section{Additional file 2}

Recombination rates of sex-averaged map. Figures of recombination rate patterns of all autosomes of the GENDISCAN and Rutgers v. 2 sexaveraged maps.

Click here for file

[http://www.biomedcentral.com/content/supplementary/1471-

2164-9-554-S2.pdf]

\section{Additional file 3}

Recombination rates of female map. Figures of recombination rate patterns of all autosomes of the GENDISCAN and Rutgers $v .2$ female maps. Click here for file

[http://www.biomedcentral.com/content/supplementary/14712164-9-554-S3.pdf]

\section{Additional file 4}

Recombination rates of male map. Figures of recombination rate patterns of all autosomes of the GENDISCAN and Rutgers v.2 male maps. Click here for file

[http://www.biomedcentral.com/content/supplementary/14712164-9-554-S4.pdf]

\section{Additional file 5}

The sex averaged map of chromosome 8 with the inversion (D8S520 and D8S1759). We found a suggestive inversion on $8 p 22$. This additional file shows the genetic map of chromosome 8 , which reflects the inversion of genetic marker order on 8p22 (D8S520 and D8S1759). The genetic length of chromosome 8 decreased more than the original map. Click here for file

[http://www.biomedcentral.com/content/supplementary/14712164-9-554-S5.xls]

\section{Acknowledgements}

We wish to thank all the subjects who participated in this study by providing their DNA and family information. We thank Dr. Bayasgalan Gombojav at Seoul National University for helpful discussions during the survey and genetic mapping. We also thank Jennifer $\mathrm{H}$. Kim at University of California, Berkeley, California, USA, and Dr. Joann Mudge at the National Center for Genome Resources, New Mexico, USA, for their kind revision of the English in the manuscript. We appreciate the assistance of the Korean Ministry of Education, Science and Technology, (Grant Number MI030503000I08N0503-00 I I0) which approved and supported the pilot study, as well as the phase I study.

\section{References}

I. Daw EW, Thompson EA, Wijsman EM: Bias in multipoint linkage analysis arising from map misspecification. Genet Epidemiol 2000, 19(4):366-380.

2. Botstein D, White RL, Skolnick M, Davis RW: Construction of a genetic linkage map in man using restriction fragment length polymorphisms. Am J Hum Genet 1980, 32(3):314-33I.

3. Dib C, Faure S, Fizames C, Samson D, Drouot N, Vignal A, Millasseau P, Marc S, Hazan J, Seboun E, et al.: A comprehensive genetic map of the human genome based on 5,264 microsatellites. Nature 1996, 380(6570): $152-154$.

4. Broman KW, Murray JC, Sheffield VC, White RL, Weber JL: Comprehensive human genetic maps: individual and sex-specific variation in recombination. Am J Hum Genet 1998, 63(3):86I-869.

5. Dausset J, Cann H, Cohen D, Lathrop M, Lalouel JM, White R: Centre d'etude du polymorphisme humain (CEPH): collabora- 
tive genetic mapping of the human genome. Genomics 1990 , 6(3):575-577.

6. Kong A, Gudbjartsson DF, Sainz J, Jonsdottir GM, Gudjonsson SA, Richardsson B, Sigurdardottir S, Barnard J, Hallbeck B, Masson G, et al: A high-resolution recombination map of the human genome. Nat Genet 2002, 3 I(3):24I-247.

7. Kong X, Murphy K, Raj T, He C, White PS, Matise TC: A combined linkage-physical map of the human genome. Am J Hum Genet 2004, 75(6): | | 43-| | 48.

8. Matise TC, Chen F, Chen W, De La Vega FM, Hansen M, He C, Hyland FC, Kennedy GC, Kong X, Murray SS, et al.: A second-generation combined linkage physical map of the human genome. Genome Res 2007, I 7(12): 1783-1786.

9. Duffy DL: An integrated genetic map for linkage analysis. Behav Genet 2006, 36(I):4-6.

10. Nievergelt CM, Smith DW, Kohlenberg JB, Schork NJ: Large-scale integration of human genetic and physical maps. Genome Res 2004, I 4(6): II99-1205.

II. Jorgenson E, Tang H, Gadde M, Province M, Leppert M, Kardia S, Schork N, Cooper R, Rao DC, Boerwinkle E, et al.: Ethnicity and human genetic linkage maps. Am J Hum Genet 2005, 76(2):276-290.

12. Goring HH, Terwilliger JD: Linkage analysis in the presence of errors III: marker loci and their map as nuisance parameters. Am J Hum Genet 2000, 66(4): 1298-1309.

13. Weitkamp LR: Proceedings: Population differences in meiotic recombination frequency between loci on chromosome $I$. Cytogenet Cell Genet 1974, I3(I): I79-182.

14. Lander ES, Green P: Construction of multilocus genetic linkage maps in humans. Proc Natl Acad Sci USA 1987, 84(8):2363-2367.

15. Cavalli-Sforza LL, Menozzi P, Piazza A: The history and geography of human genes. Princeton NJ: Princeton University Press; 1994.

16. Cavalli-Sforza LL, Feldman MW: The application of molecular genetic approaches to the study of human evolution. Nat Genet 2003, 33(Suppl):266-275.

17. Egel R: Recombination and meiosis: crossing-over and disjunction. Berlin; New York: Springer; 2008.

18. Guthery SL, Salisbury BA, Pungliya MS, Stephens JC, Bamshad M: The structure of common genetic variation in United States populations. Am J Hum Genet 2007, 8 I (6): |22 I-I23I.

19. Gabriel SB, Schaffner SF, Nguyen H, Moore JM, Roy J, Blumenstiel B, Higgins J, DeFelice M, Lochner A, Faggart M, et al.: The structure of haplotype blocks in the human genome. Science 2002, 296(5576):2225-2229.

20. Nozawa M, Kawahara Y, Nei M: Genomic drift and copy number variation of sensory receptor genes in humans. Proc Natl Acad Sci USA 2007, 104(5I):2042I-20426.

21. McVean GA, Myers SR, Hunt S, Deloukas P, Bentley DR, Donnelly P: The fine-scale structure of recombination rate variation in the human genome. Science 2004, 304(5670):58I-584.

22. Myers S, Bottolo L, Freeman C, McVean G, Donnelly P: A fine-scale map of recombination rates and hotspots across the human genome. Science 2005, 310(5746):32I-324.

23. Crawford DC, Bhangale T, Li N, Hellenthal G, Rieder MJ, Nickerson $D A$, Stephens M: Evidence for substantial fine-scale variation in recombination rates across the human genome. Nat Genet 2004, 36(7):700-706.

24. Graffelman J, Balding DJ, Gonzalez-Neira A, Bertranpetit J: Variation in estimated recombination rates across human populations. Hum Genet 2007, I 22(3-4):30I-310.

25. Ziegler A, König IR: A statistical approach to genetic epidemiology: concepts and applications. Weinheim: Wiley- $\mathrm{VCH} ; 2006$.

26. McPeek MS, Sun L: Statistical tests for detection of misspecified relationships by use of genome-screen data. Am J Hum Genet 2000, 66(3): 1076-1094.

27. O'Connell JR, Weeks DE: PedCheck: a program for identification of genotype incompatibilities in linkage analysis. $\mathrm{Am} J$ Hum Genet 1998, 63(1):259-266.

28. Weeks DE, Sobel E, O'Connell JR, Lange K: Computer programs for multilocus haplotyping of general pedigrees. Am J Hum Genet 1995, 56(6): 1506-1507.

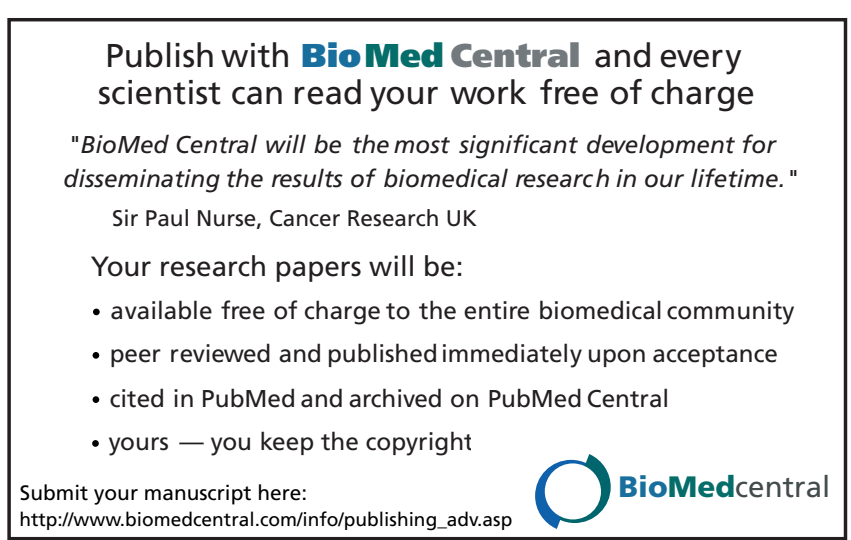

